\title{
HEAT TRANSFER IN SEPARATED FLOWS ON THE PRESSURE SIDE OF TURBINE BLADES
}

\author{
P. De La Calzada ${ }^{1,2}$, M. Valdes ${ }^{2}$, and M. A. Burgos ${ }^{3}$ \\ IIndustria de TurboPropulsores S. A., Madrid, Spain \\ ${ }^{2}$ Polytechnic University of Madrid, UPM, Madrid, Spain \\ ${ }^{3}$ Polytechnic University of Cartagena, UPCT, Cartagena, Spain
}

\begin{abstract}
Heat transfer in separated flows on the pressure side of a typical high lift turbine profile is numerically investigated by means of an in-house CFD code. The numerical code was first validated on attached flows in turbine blades. To obtain flow separation cases, the profile is subject to large negative incidences so that a separation bubble is obtained at the pressure side. The numerical results are compared to available experimental data for code validation. It is shown how local minima and maxima values of the heat transfer coefficient are related to the separation and reattachment points, where the velocity component perpendicular to the wall is shown to have a significant effect on the heat transfer.
\end{abstract}

\section{INTRODUCTION}

The increasing demand of more efficient gas turbine engines is further stressing the physical understanding of aerothermal phenomena occurring in turbines. Turbine inlet temperature (TIT) has increased rapidly in the last decades enabled by the extensive use of increasingly effective cooling technologies. Further reductions in weight and cost targets have also required the development of thin low pressure turbine (LPT) profiles where flow separation might occur at the pressure side even at design conditions. These operating conditions of LPT have introduced new challenges in terms of understanding the aerothermal phenomena, as well as in the development of simulation tools able to predict these phenomena. In particular, the accurate prediction of thermal effects due to separation and reattachment of the flow on LPT profiles is an important new requirement.

The use of numerical prediction methods (i.e., CFD), which have acquired an acceptable level of maturity, becomes essential for the aerothermal design of turbomachinery components. Traditionally, these tools have been validated for heat transfer in high pressure turbines (HPT) featuring high Reynolds number and attached flow all along the profile. In the last decade, the capability of different CFD codes 


\begin{tabular}{|c|c|c|c|}
\hline \multicolumn{4}{|c|}{ NOMENCLATURE } \\
\hline Cax & axial chord (m) & $u, U$ & velocity, $\mathrm{m} / \mathrm{s}$ \\
\hline$C f$ & skin-friction coefficient & VKI & von Karman Institute \\
\hline CFD & computational fluid dynamics & $x$ & real chord axis coordinate, $\mathrm{m}$ \\
\hline $\mathrm{Ch}$ & Stanton number & $y^{+}$ & nondimensional distance from the \\
\hline$C p$ & pressure coefficient $\frac{2\left(P-P_{\infty}\right)}{\rho_{\infty} \mu_{\infty}}$, specific & $(\partial / \partial n)_{n}$ & $\begin{array}{l}\text { wall } \\
\text { gradient normal to the wall }\end{array}$ \\
\hline $\mathrm{HTC}$ & $\begin{array}{l}\mathrm{W} / \mathrm{m}^{2} \mathrm{~K}\end{array}$ & $\beta$ s & $\begin{array}{l}\text { stagger angle } \\
\text { flow angle }\end{array}$ \\
\hline HPT & high pressure turbine & $\delta$ & boundary layer thickness \\
\hline$k$ & $\begin{array}{l}\text { thermal conductivity of the fluid, } \\
\mathrm{W} / \mathrm{mK}\end{array}$ & $\begin{array}{l}K \\
\mu\end{array}$ & $\begin{array}{l}\text { turbulent kinetic energy, } \mathrm{m}^{2} / \mathrm{s}^{2} \\
\text { viscosity, } \mathrm{kg} / \mathrm{m}-\mathrm{s}\end{array}$ \\
\hline$L$ & $\begin{array}{l}\text { W/mK } \\
\text { real chord, } \mathrm{m}\end{array}$ & $\rho$ & density, $\mathrm{kg} / \mathrm{m}^{3}$ \\
\hline LPT & low pressure turbine & $\tau$ & shear stress \\
\hline $\mathrm{M}$ & Mach number & $\omega$ & specific dissipation rate \\
\hline$M U^{2} \mathrm{~S}^{2} \mathrm{~T}$ & multirow unsteady unstructured & $\infty$ & free-stream conditions \\
\hline & specific solver for turbomachinery & $o$ & stagnation conditions \\
\hline$P$ & pressure $\left(\mathrm{N} / \mathrm{m}^{2}\right)$ & des & design conditions \\
\hline $\operatorname{Pr}$ & Prandtl number & $e$ & boundary layer edge conditions \\
\hline RANS & $\begin{array}{l}\text { Reynolds-Averaged Navier Stokes } \\
\text { equations }\end{array}$ & $\begin{array}{l}1 \\
2\end{array}$ & $\begin{array}{l}\text { inlet conditions } \\
\text { exit conditions }\end{array}$ \\
\hline $\operatorname{Re}$ & Reynolds number & $i s$ & isentropic conditions \\
\hline$T$ & temperature, ${ }^{\circ} \mathrm{K}$ & $s$ & static conditions \\
\hline$t / L$ & pitch ratio & $w$ & wall conditions \\
\hline TIT & turbine inlet temperature & $X$ & local conditions \\
\hline
\end{tabular}

to predict the heat transfer on these configurations has been investigated focusing mainly in analysing the mesh sensitivity and turbulence model effects (e.g., Tutar and Sönmez [1] or El-Batsh [2] among others). The cases of small local flow separation at the suction side of LPT airfoils with sufficiently low Reynolds number and high diffusion have been also extensively investigated. However, the main focus of these cases is on the aerodynamic behavior, being very few the works focused on the details of the heat transfer physics (e.g., Schobeiri et al. [3]).

Much attention has been paid to the investigation of large flow separation in simple cases, including both velocity related measurements and heat transfer measurements. These include experimental investigations of backward-facing steps as Vogel and Eaton [4] or Sparrow et al. [5], where the relationship between the separation region and the heat transfer features was studied. Corresponding numerical investigations have been performed on similar configurations like the one by Kaminejad et al. [6] where only laminar conditions and very low Reynolds numbers are considered. The effect of turbulence was taken into account for example by Rhee and Sung [7], where good agreement with experimental data was also found for very low Reynolds numbers. More recently, Rhee and Sung [8] also investigated the effect of local forcing on the separation and reattaching flow.

However, very little attention has been paid to the heat transfer in large separated flow regions in turbine representative conditions. Bassi et al. [9] present CFD results on the separated flow region of a HPT airfoil with cutted trailing edge with no cooling ejection, but only a short discussion about the separated flow physics is 
included. Regarding experimental investigations, Rivir et al. [10] have measured the flat plate heat transfer in a region of turbulent separation, and Bellows and Mayle [11] have measured the heat transfer on a blunt body leading edge separation bubble both for cases of high Reynolds number. More recently, De la Calzada and Alonso [12] performed a numerical investigation of large flow separation region at the pressure side of a turbine profile but not comparison with experimental results was included. Lutum and Cottier [13] presented a similar investigation, but results indicated that simulations were not able to reproduce experimental heat transfer results at the pressure side separation region especially for low turbulence levels.

From experimental investigations, it is known qualitatively that separated flow regions are characterised by large and rapid variations of the heat transfer (e.g., Rhee and Sung [8]). Furthermore, the heat transfer presents a local minimum and a local maximum in the vicinity of separation and reattachment points respectively, with regions where the heat transfer coefficient (HTC) is much larger than that of attached flows $[10,14]$. Taking into account that separated flow regions are usually characterized by high turbulence levels and large scale unsteadiness, there is a tendency in the heat transfer community to explain the heat transfer phenomena in separated flows in terms of the generation of turbulence rather than in terms of the dynamic and thermal boundary layers relationship.

The aim of the present investigation is to perform a detailed numerical study of the heat transfer phenomena in separated flows at flow conditions representative of LPT airfoils. A comparison with experimental data from the European programme AITEB [15] is performed, hence allowing the validation of the code and the confirmation of the main flow features. Based on these numeric results, an attempt is made to understand and explain the flow physics in the vicinity of separation and reattachment points that can influence the heat flux. In particular, the relationship between the dynamic and thermal boundary layers and the importance of the velocity component perpendicular to the wall in creating injection of flow towards the wall or ejection of flow from the wall and their effect in the heat transfer is analyzed.

\section{CFD SIMULATIONS AND VALIDATION}

\subsection{CFD Modeling}

In the present investigation, an in-house CFD code named $\mathrm{MU}^{2} \mathrm{~S}^{2} \mathrm{~T}$ is used for calculations. This code solves the RANS equations written in conservative form in an absolute frame of reference. The scheme used is based upon Jameson et al. [16]. Convective terms are discretized using a cell vertex scheme, and the viscous terms are computed by means of the Hessian matrix. Integration in time is performed using an explicit five stage Runge-Kutta scheme where the viscous and artificial dissipation terms are evaluated in the first, third, and fifth stages. The code runs on unstructured meshes which are built by a quasi-structured layer all along the walls, where viscous effects are expected to be dominant and by a fully triangular unstructured mesh in the rest of the flow domain obtained by Steiner triangulation [17]. For turbulence simulation, the two equations $k-0$ model from Wilcox [18] is implemented. More details of the numerical code can be found in Corral and Contreras [19]. 
Numerical results are post processed to obtain heat and mass transfer relevant parameters at the walls. This is performed by computing velocity and temperature variation in the direction perpendicular to the wall so that heat transfer and friction coefficient as well as Stanton number can be computed, as defined in Eqs. (1) and (2). The local Stanton number is the equivalent for the temperature to the local skin-friction coefficient for the velocity. Although the local Stanton number variations do not represent variations in heat flux alone but also take into account the local velocity value, it is the most adequate parameter to describe the thermal boundary layer behavior and to develop special correlations for heat transfer estimation (i.e., Reynolds-Colburn analogy).

$$
\begin{gathered}
\mathrm{Ch}_{x}=\frac{\mathrm{HTC}_{x}}{\rho_{e} c_{p} u_{e}}=\frac{-k\left(\frac{\partial T}{\partial n}\right)_{w}}{\left(T_{o}-T_{w}\right) \rho_{e} c_{p} u_{e}} \\
C f_{x}=\frac{\tau_{w}}{\frac{1}{2} \rho_{e} u_{e}^{2}}=\frac{\mu\left(\frac{\hat{\partial} u}{\partial n}\right)_{w}}{\frac{1}{2} \rho_{e} u_{e}^{2}}
\end{gathered}
$$

Note, that the total temperature is used in the definitions instead of the adiabatic wall temperature, even though compressible effects and therefore viscous dissipation may be important since the representative cases for LPT usually imply an exit Mach number of around 0.5 , as we have in our study. However, the difference between the aforementioned coefficients and the corresponding compressible definitions can be kept sufficiently low if the wall temperature for the computations is properly chosen. In our particular cases, the total temperature is defined as in the experiments and the wall temperature is taken around $25 \mathrm{~K}$ higher than the fluid temperature, which keeps the difference between compressible and incompressible heat and mass transfer coefficient values lower than $2 \%$ even at regions with Mach numbers around 0.5 . This wall temperature value also develops a thermal boundary layer whose magnitude is large enough to avoid high sensitivity to any random numerical errors in the resolution of the temperature field around the wall.

\section{2. $\mathrm{MU}^{2} \mathrm{~S}^{2} \mathrm{~T}$ Validation}

$\mathrm{MU}^{2} \mathrm{~S}^{2} \mathrm{~T}$ is validated for heat transfer in attached flows against a highly loaded transonic turbine guide vane named LS89 widely used for validation purposes [20].

All grids were generated with G2D, an in-house tool for unstructured mesh generation on turbomachinery. The computational grid around the profile was generated following the unstructured and quasi-structure approach mentioned above. Figure 1 shows detail of the mesh in the external region. A growing mesh rate is specified on the homogeneous triangular mesh when moving from the profile towards the inlet boundary. Also, the quasi-structured region all around the profile and wake with stretched triangles following the main direction of the flow can be observed.

First, a CFD model with a Reynolds number equal to $10^{6}$ and an isentropic exit Mach number of 0.875 was run to validate the pressure distribution around 

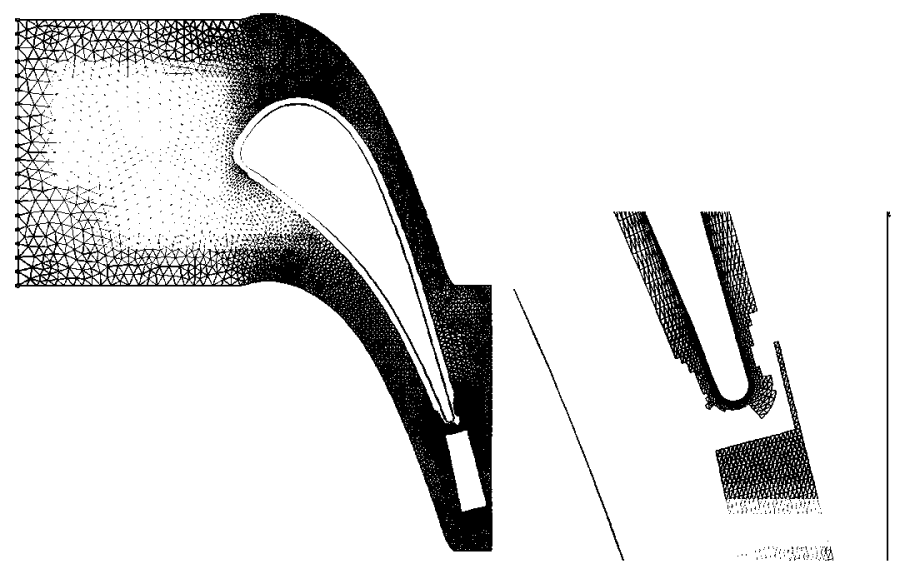

Figure 1. Unstructured and quasi-structured meshes around the LS89 turbine profile.

the blade. Excellent agreement with the experimental results was achieved, as shown in Figure 2.

Second, for heat transfer validation purposes only laminar simulations were performed, since we are not especially concerned about turbulent effects in this investigation. In particular, one case with similar Reynolds number to the one found in LPT (i.e., $5 \times 10^{5}$ ) was chosen where no transition of the boundary layer was observed in the experiments. A constant stagnation temperature of $420 \mathrm{~K}$ and a constant wall temperature of $450 \mathrm{~K}$ were specified.

When obtaining heat transfer results from these computations it was noted that some wiggles were appearing, especially in the leading edge and suction side surface as plotted in Figure 3. These are thought to be due to some truncation errors in the specification of geometry points, as other authors like Gehrer and Jericha [21] and

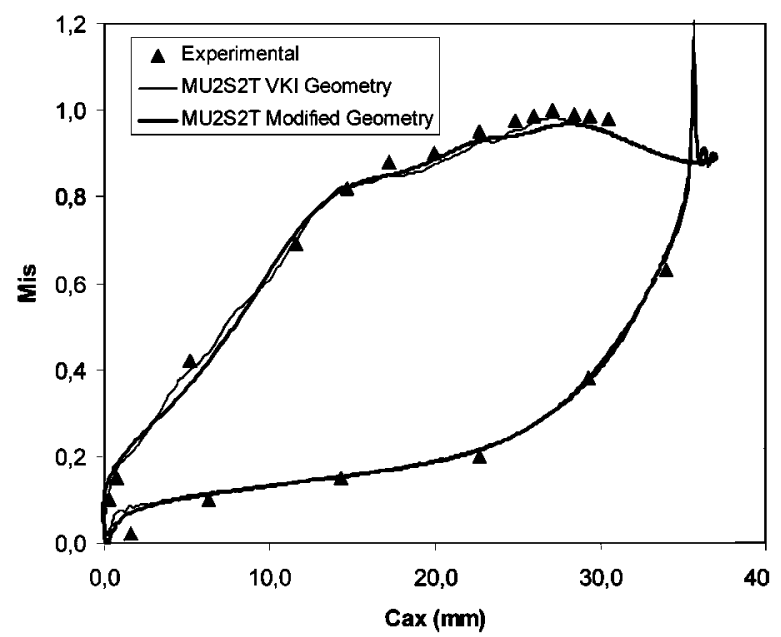

Figure 2. LS89 blade isentropic Mach number distribution. ( $\operatorname{Re}_{2 i s}=10^{6}$ and $\mathrm{M}_{2 i s}=0.875$.) 


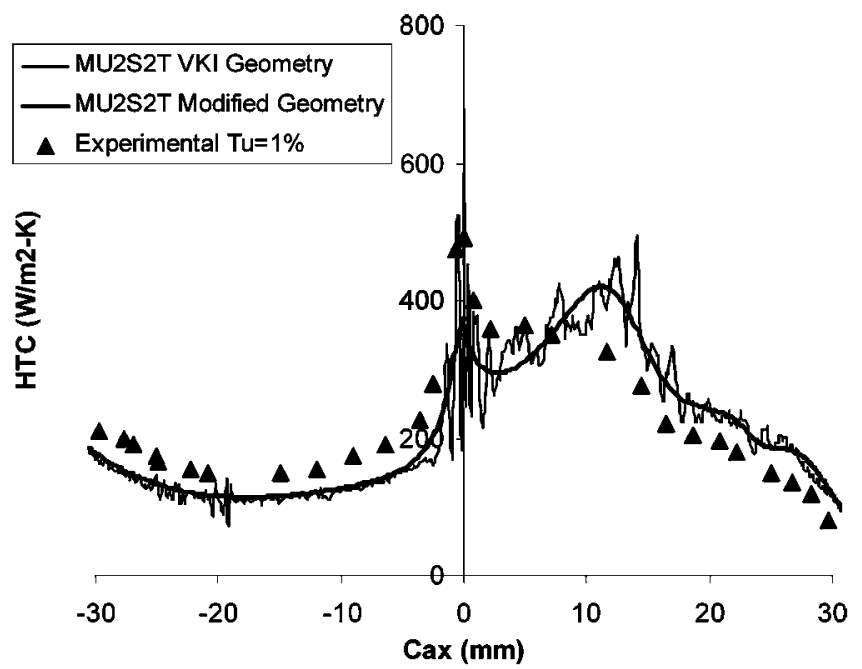

Figure 3. LS89 Blade heat transfer distribution. $\left(\mathrm{Re}_{2 i s}=5.0 \times 10^{5}, \mathrm{M}_{2 i s}=0.92\right.$, and $T_{w}=450^{\circ} \mathrm{K}$.)

Boyle and Ameri [22] have reported. In order to solve such problem; a redesign of the geometry was performed while keeping the same pressure distribution and global geometry constraints. The ensured continuity of curvature in the new geometry had a strong impact on the results, and then smooth variation on the HTC distribution were obtained as plotted also in Figure 3. However, some discrepancies with the measured data in the suction side acceleration region were obtained, especially in a region around $1 / 3$ of the axial chord (which seems to be a common problem based on similar results obtained by Tutar and Sönmez [1]).

Regarding grid sensitivity, some different cases were computed to obtain the optimum $y^{+}$range in cells adjacent to the wall in order to accurately predict the heat transfer features. As a result of this study, the optimum range for heat transfer calculations was found to be $y^{+}<3$, even in laminar regions.

\subsection{Description of the T106-300 Model, Computational Grids, and Results for Attached Flow}

The T106-300 blade section has been used as a generic geometry representative of a typical highly loaded LPT airfoil [23] (see Figure 4 for cascade geometry and conditions details). In this investigation, the T106 blade profile is subject to extremely large negative incidences in order to have a large separation bubble on the pressure side. Mach and Reynolds numbers are varied around typical LPT values.

The generated grid is hybrid in nature with higher definition in regions adjacent to the wall, trailing edge and leading edge, as shown in Figure 5. Due to the expected flow separation at the pressure side when the profile is subject to high negative incidence, on this investigation the viscous mesh is extended to a region larger than attached flows would require for this Reynolds number. The objective of this large region with high definition is to capture the shear layers and flow features on the 


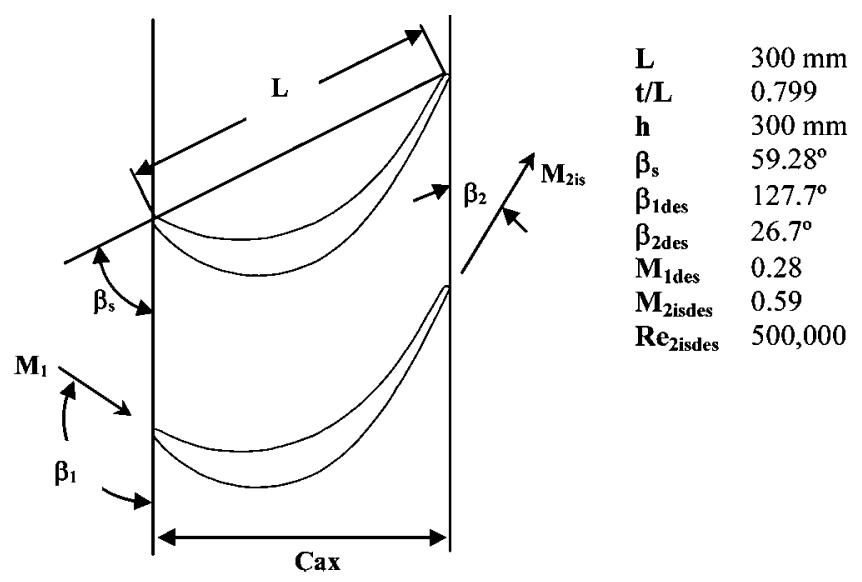

Figure 4. T106-300 Cascade geometry and aerodynamic design conditions.

pressure side large bubble. However, in order to avoid any mesh sensitivity the same grid consisting of 8,623 nodes was kept unchanged for all cases, including the attached flow achieving a range of $y^{+}$values at the pressure side in the order of $y^{+}<3$.

Results at design conditions are shown in Figures 6 and 7. Pressure distribution is considered to match well with the experiments, in particular on the major part of the suction side. Since we are interested mainly in the flow along the pressure side, no attention will be paid to the separation bubble at the back suction surface that the code does not predict probably due to a too soon turbulence generation and boundary layer transition. Although the level of pressure achieved by the numerical results at the pressure side of the profile is lower than the experimental data, the heat

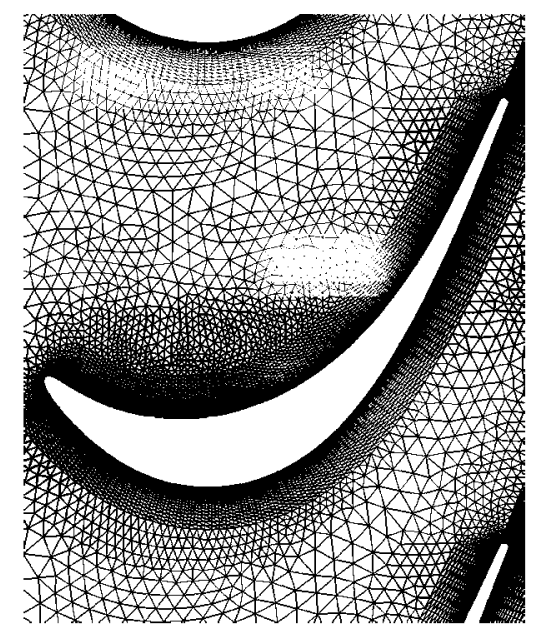

Figure 5. 2-D hybrid mesh around the T106 blade. 


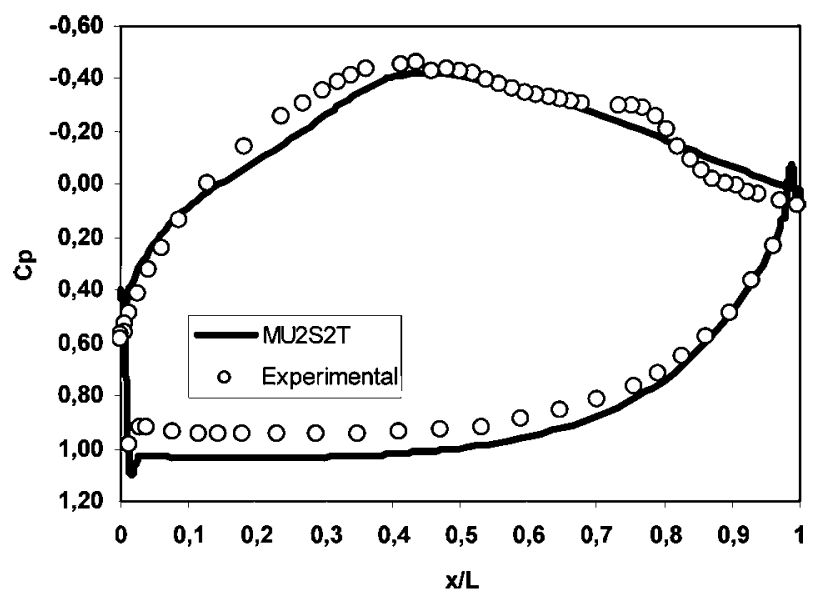

Figure 6. T106 profile pressure coefficient at design conditions. No separation. $\left(\operatorname{Re}_{2 i s}=1.5 \times 10^{5}\right.$, $\mathrm{M}_{2 i s}=0.5, \beta_{1}=127.7^{\circ}$, and $T_{w}=325^{\circ} \mathrm{K}$.)

transfer level matches well with the experiments. However, more HTC oscillations are found in the experiments compared with the smoother results predicted by the numerical simulation. It is interesting to note that the heat transfer measurements at the acceleration region of the leading edge decrease to lower values than the CFD results. This might indicate that the profile is subject to a slightly larger negative incidence in the experiments, hence creating an acceleration-deceleration behavior achieving a higher final pressure as shown by the results. The final acceleration region towards the trailing edge has a more pronounced effect on the numerical simulation, where the heat transfer value shows higher increase due to the expected thinning of the boundary layer with the increase in the external velocity.

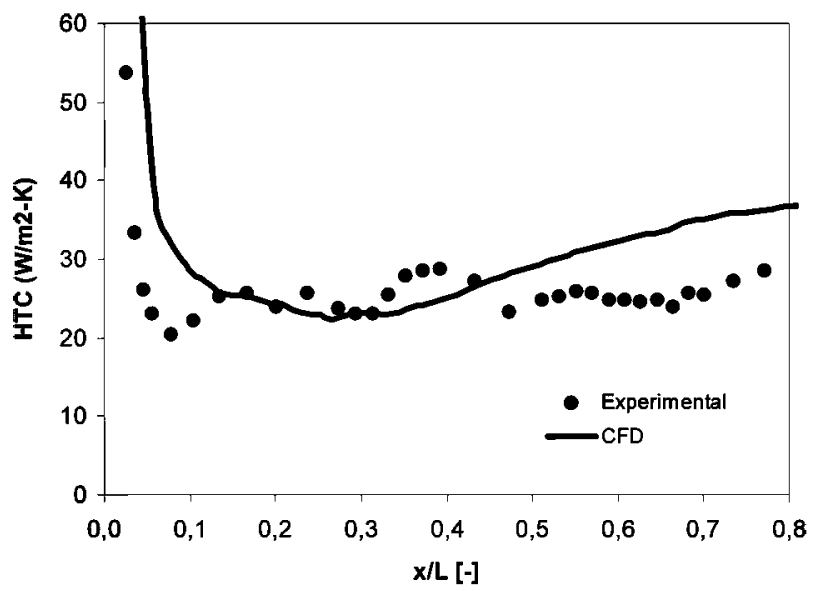

Figure 7. T106 heat transfer results on the pressure side at design conditions. $\left(\operatorname{Re}_{2 i s}=1.5 \times 10^{5}, \mathrm{M}_{2 i s}=0.5\right.$, $\beta_{1}=127.7^{\circ}$, and $T_{w}=325^{\circ} \mathrm{K}$.) 


\section{SEPARATED FLOW RESULTS}

\subsection{Large Separation Bubble}

Results at the extreme conditions of $-37.7^{\circ}$ negative incidence (i.e., inlet angle $\beta_{1}=90^{\circ}$ ) are shown first. The comparison between numerical and experimental results in terms of pressure distribution along the airfoil is presented in Figure 8. The separation region is characterized by low velocities and a fairly constant pressure distribution. However, at the reattachment region the static pressure increases reaching a local maximum (i.e., local minimum value of $C p$ in Figure 8), which indeed indicates the reattachment point. The experimental results indicate, to some extent, a shorter separation bubble which reattaches earlier, hence starting earlier also the acceleration towards the trailing edge. This may be related to the already identified higher pressure at the pressure side predicted by the CFD, which might indicate some slight difference in local incidence angle between the experiments and the simulations.

Some more detail about the flow can be identified by comparing experimental and numerical heat transfer results shown in Figure 9. Both experimental and numerical results show two local minima and maxima between the extreme values achieved at leading and trailing edges (these extreme values are not shown in the graph). The first minimum occurring at around $0.03 x / L$ corresponds to the expected reduction in heat transfer rate at the separation point. The maximum occurring at around $0.6 x / L$ corresponds to the expected increase in heat transfer rate at the reattachment point. However, a second local maximum at around $0.1 x / L$ and a second local minimum at around $0.2-0.3 x / L$ (depending if looking at experimental or numerical results) occur, hence indicating some internal structure on the separation bubble that produces relevant changes in the heat transfer features. Although the extension of the separation bubble is somehow smaller in the experiment compared with the simulations, the trend and levels in both cases confirm the same physical behaviour.

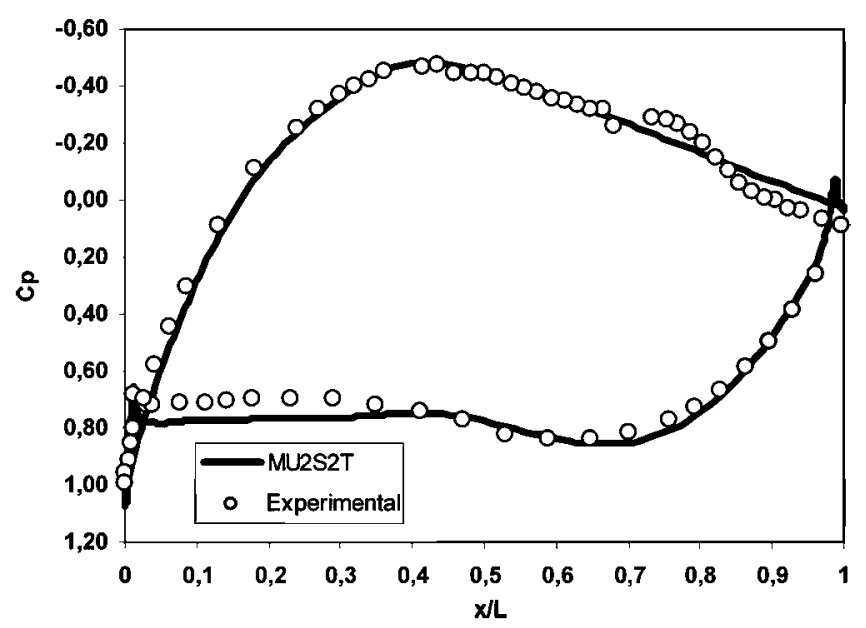

Figure 8. T106 profile pressure coefficient. ( $\operatorname{Re}_{2 i s}=1.5 \times 10^{5}, \mathrm{M}_{2 i s}=0.5, \beta_{1}=90^{\circ}$, and $T_{w}=325^{\circ} \mathrm{K}$.) 


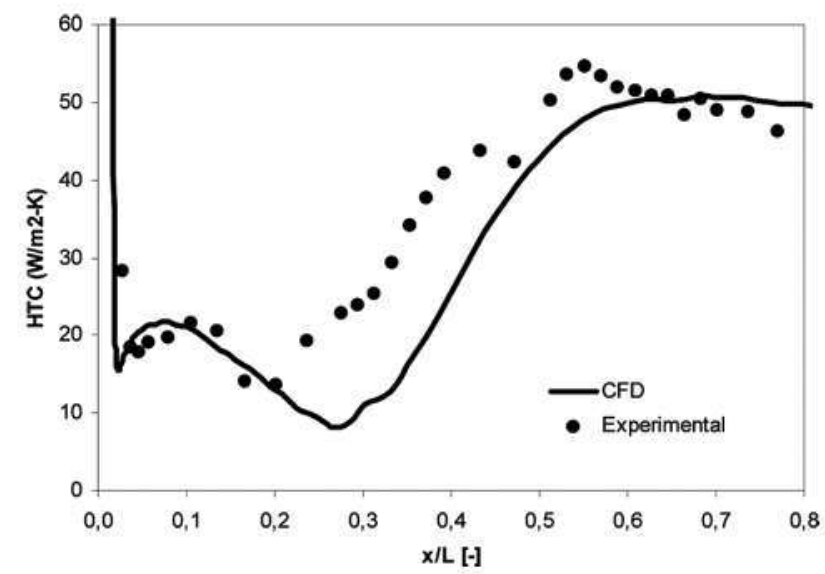

Figure 9. T106 heat transfer coefficient. $\left(\operatorname{Re}_{2 i s}=1.5 \times 10^{5}, \mathrm{M}_{2 i s}=0.5, \beta_{1}=90^{\circ}\right.$, and $T_{w}=325^{\circ} \mathrm{K}$.)

In Figure 10 the Mach number and total pressure fields are plotted where the large bubble at the pressure side can be clearly identified. Helped by the streamlines traces, the multiple bubble configuration can be also identified. In this particular case, two bubbles appear. As confirmed in Figure 11 by the velocity vectors, one small bubble is stretched towards the pressure wall, developing at the center of the full separation region whose vortex is rotating counterclockwise, and one large bubble, rotating clockwise is extending up to the external shear layer along the major part of the pressure side, having its vortex core at the rear part of the separation region while extending its vortex influence also to the front part.

Detail of the temperature field and flow velocities in the regions of flow separation and reattachment are plotted in Figures 11 and 12. At points 1 and 3, the flow is separating and a large component of the velocity perpendicular and directed away
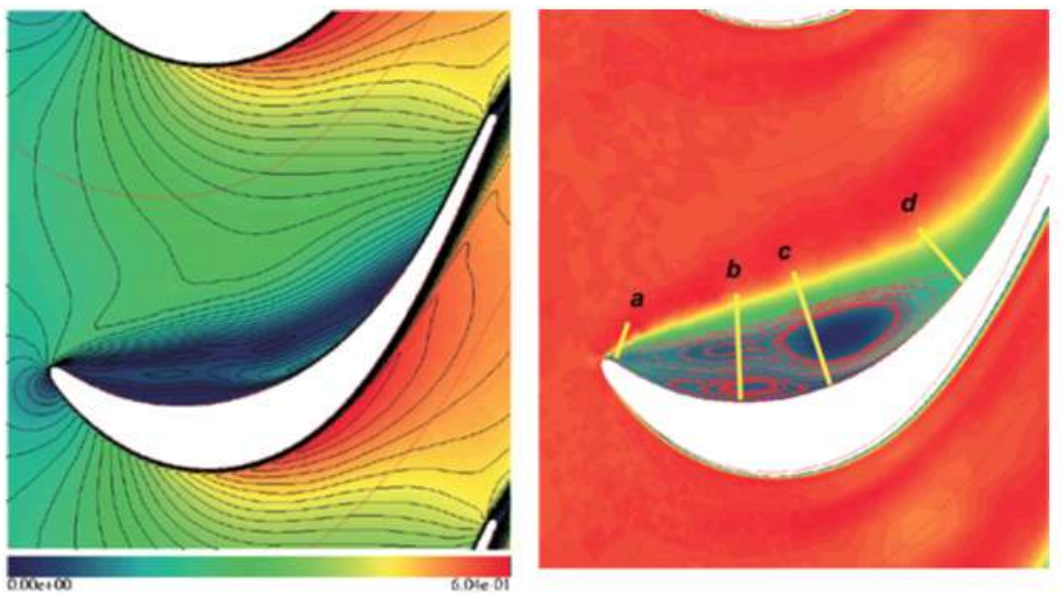

Figure 10. Mach field (left) and total pressure field with bubble streamlines (right) $\left(\operatorname{Re}_{2 i s}=1.5 \times 10^{5}\right.$, $\mathrm{M}_{2 i s}=0.5, \beta_{1}=90^{\circ}$, and $T_{w}=325^{\circ} \mathrm{K}$.) (color figure available online). 

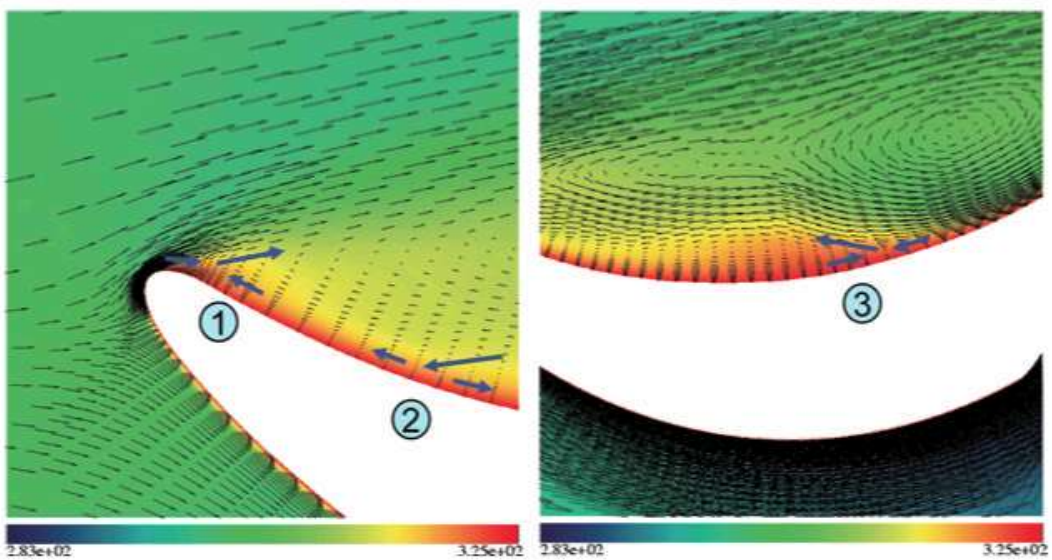

(3)

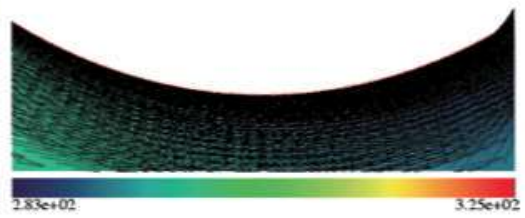

Figure 11. Temperature field and velocity vectors at the front and middle parts of the separation bubble 1. Ejection stagnation region at separation region; 2 . impingement stagnation region due to an internal bubble; and 3. ejection stagnation region due to the internal bubble separation. $\left(\operatorname{Re}_{2 i s}=1.5 \times 10^{5}\right.$, $\mathrm{M}_{2 \text { is }}=0.5, \beta_{1}=90^{\circ}$, and $T_{w}=325^{\circ} \mathrm{K}$.) (color figure available online).

from the wall exists. This flow configuration takes heat from the side walls and ejects it, creating an ejection stagnation or fountain-like region where the wall thermal field is penetrating the main flow helped by the perpendicular component of the velocity, hence increasing the effective thermal boundary layer and decreasing the heat transfer rate. This phenomena is particularly clear in front of point 3 , where the increase in the thermal boundary layer thickness can be easily identified by the extension of the high temperature region close to the wall in Figure 11. Point 3 corresponds to the

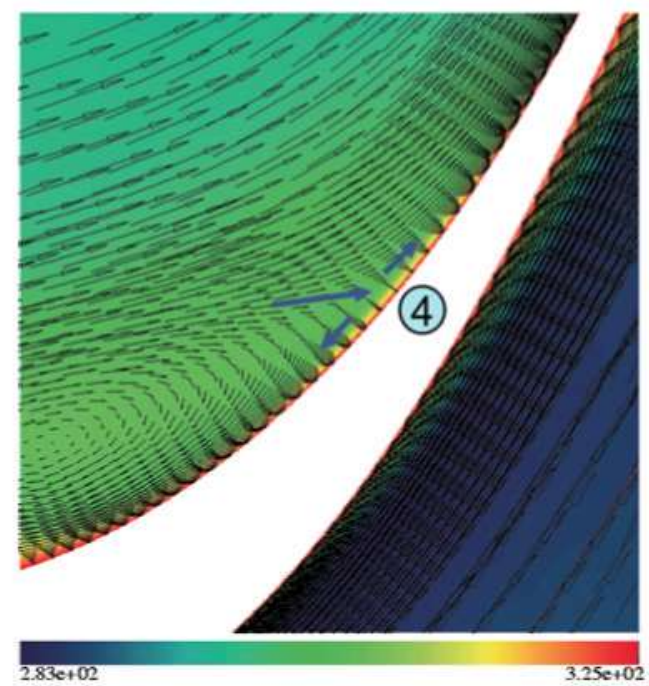

Figure 12. Temperature field and velocity vectors at the rear part of the separation region 4 . Injection stagnation region at main bubble reattachment point. $\left(\operatorname{Re}_{2 i s}=1.5 \times 10^{5}, \mathrm{M}_{2 i s}=0.5, \beta_{1}=90^{\circ}\right.$, and $T_{w}=325^{\circ} \mathrm{K}$.) (color figure available online). 
separation of an internal second bubble, which must also exist in the experiment configuration since the local minimum can be also identified in measurements in Figure 9. Points 2 and 4 correspond to reattachment points where there is an important component of the velocity perpendicular and towards the wall, hence taking fresh fluid to the wall and creating an injection stagnation region where the thermal boundary layer is reduced and heat transfer is increased.

To further investigate the thermal boundary layer developing through the separation region the temperature profiles developing along straight lines perpendicular to the wall, marked a-d in Figure 10, are plotted in Figure 13. Note, that the $y$ coordinate is nondimensionalized with the thermal boundary layer thickness, which is basically coincident with the thickness of the separated region. Dotted lines represent the temperature distribution of the corresponding adiabatic wall case where static temperature only varies as a result of the velocity profile (being the stagnation temperature fundamentally constant), while solid lines show the temperature distribution within the re-circulation region at different distances from the wall in the case with heat transfer and heated wall. The lowest wall temperature gradient is obtained at the leading edge separation point 1 (line a), where even with the thermal boundary layer being relatively thin the fluid temperature shows a low gradient specially close to the wall driven by the ejection of heated flow from the wall through the ejection stagnation region configuration. On the contrary, the highest heat flux is achieved at the main bubble reattachment point 4 (line d) where, additionally to the thin thermal boundary layer thickness, the fluid temperature variation is mainly concentrated at the wall in a region about $10 \%$ of that thickness, hence increasing the temperature gradient at the wall. This reduction of the effective boundary layer thickness is driven by the injection stagnation region configuration, where the

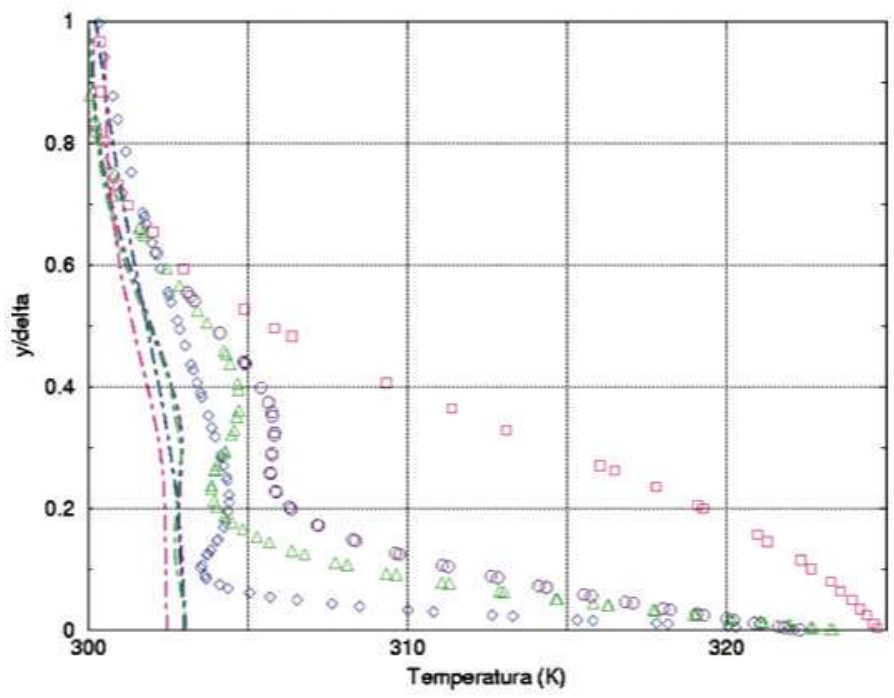

Figure 13. Thermal boundary layer on the bubble Dotted lines: adiabatic wall; symbols: heated wall. ( $\square$ ) Separation at leading edge $(a) ;(\triangle)$ reattachment region of middle bubble $(b) ;(0)$ mid-region of middle bubble $(c)$; and $(\diamond)$ reattachment of main bubble $(d)$. $\left(\operatorname{Re}_{2 i s}=1.5 \times 10^{5}, \beta_{1}=90^{\circ}, \mathrm{M}_{2 i s}=0.5\right.$, and $T_{w}=325^{\circ} \mathrm{K}$.) (color figure available online). 


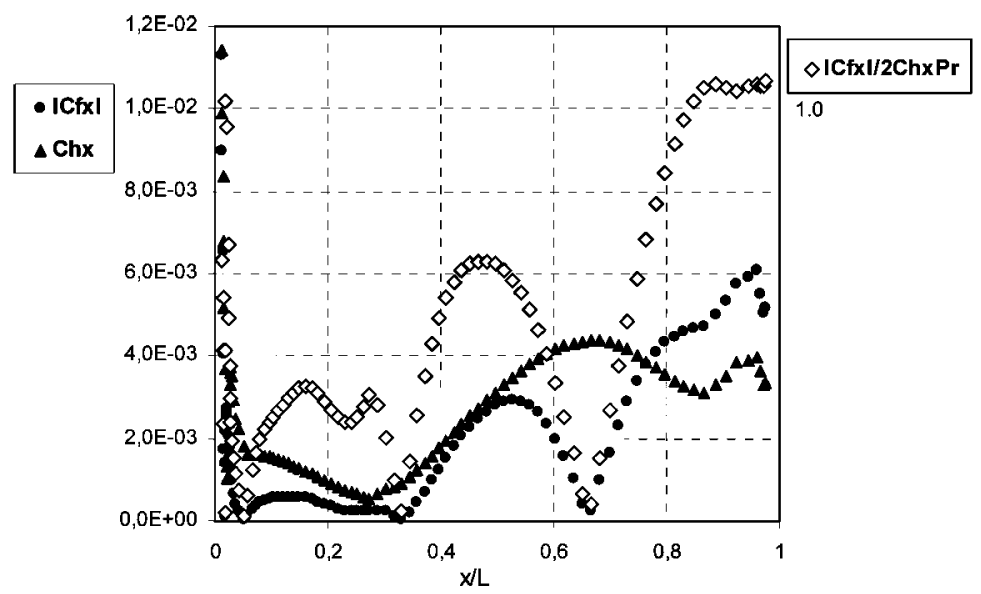

Figure 14. Reynolds-Colburn analogy. $\left(\operatorname{Re}_{2 i s}=1.5 \times 10^{5}, \mathbf{M}_{2 i s}=0.5, \beta_{1}=90^{\circ}\right.$, and $T_{w}=325^{\circ} \mathrm{K}$.)

velocity component perpendicular to the wall is forcing the thermal boundary layer to be squeezed towards the wall.

As an additional proof showing that there is low coupling between the dynamic and thermal boundary layers and their gradients in separated regions, the relationship between the velocity parallel to the wall and temperature gradients at the wall is investigated. It is widely accepted that the Reynolds-Colburn analogy is only reliable in attached flows only for modest, near-zero, pressure gradients, and with a constant wall temperature [24]. The computed local skin-friction coefficient (absolute value), the Stanton number, and the Reynolds-Colburn analogy are shown in Figure 14 to further demonstrate that the analogy between dynamic and thermal boundary layer is not valid for separated flows even when no pressure gradient exists (e.g., Spalart and Coleman [25]). Only at the rear acceleration region where attached flow is ensured, the Reynolds analogy tends to follow the correlation showing a conventional relationship between dynamic and thermal boundary layers and their gradients at the wall. Furthermore, unlike the Stanton number, the skin friction approaches zero not only at the separation and reattachment regions but also along the major part of the separated flow region, hence confirming that the ReynoldsColburn analogy is not applicable. This is clear proof that there is a very weak coupling between velocity parallel to the wall and thermal boundary layers in separated flows. On the contrary, it is the convective transport of fluid in a direction normal to the wall and the fluid conduction effects in low velocity regions what drive the heat transfer phenomenon, hence supporting once again the prime role of the stagnation region configurations on the heat transfer mechanism.

\subsection{Inlet Flow Angle Effects}

Inlet flow angles of $90^{\circ}, 100^{\circ}, 110^{\circ}$ (i.e., $-37.7,-27.7^{\circ}$, and $-10.7^{\circ}$ incidence angle, all with separated flows at pressure side), and $127.7^{\circ}$ (i.e., $0^{\circ}$ incidence angle, with pressure side attached flow) have been simulated for the nominal isentropic exit Reynolds number of $1.5 \times 10^{5}$ and an isentropic exit Mach number of 0.5 . The 


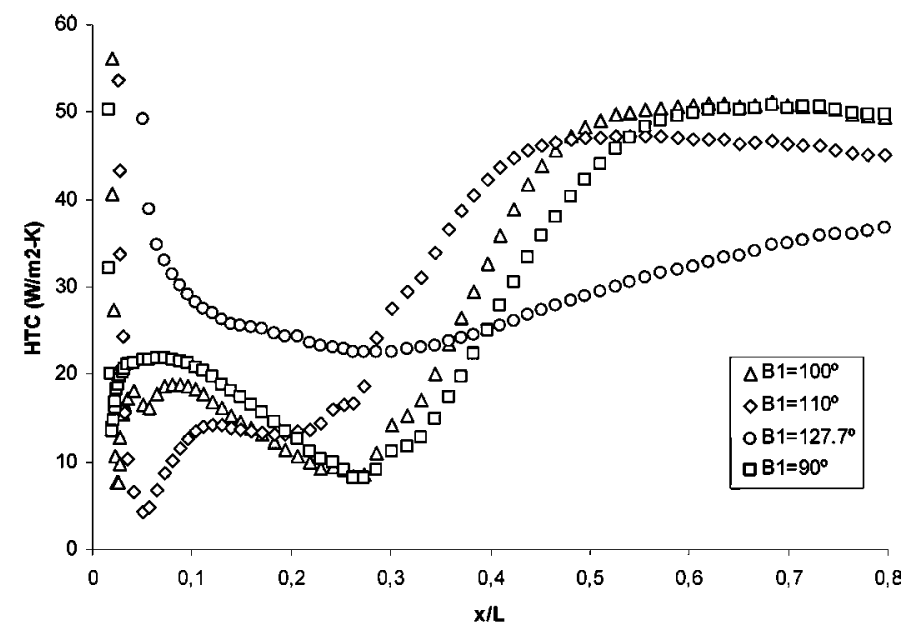

Figure 15. Heat transfer coefficient for different negative incidences. $\left(\operatorname{Re}_{2 i s}=1.5 \times 10^{5}, \mathrm{M}_{2 i s}=0.5\right.$, and $T_{w}=325^{\circ} \mathrm{K}$.)

results in terms of HTC and Stanton number are presented in Figures 15 and 16. As expected, the size of the bubble is decreasing with the reduction of the negative incidence angle as can be concluded from the location of the maximum values of Stanton numbers in Figure 16. All the separated flow cases show relatively large bubbles varying the reattachment points from $0.5 x / L$ for $-17.7^{\circ}$ incidence up to $0.6 x / L$ for $-37.7^{\circ}$ incidence. Unlike the HTC whose local maximum value at reattachment point is maintained almost constant along the attached acceleration region up to the trailing edge region, the Stanton number clearly generates a more pronounced local maximum value at the reattachment point driven by the combination of maximum heat flux and static pressure (i.e., minimum external velocity).

It is noticeable that in all separated flow cases the same multiple bubble configuration is obtained as indicated by the presence, within the separated region, of

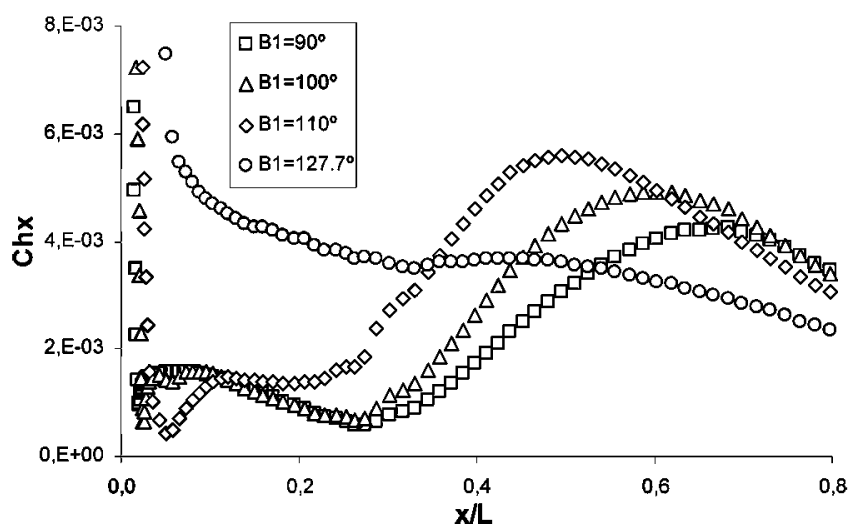

Figure 16. Stanton number for different negative incidences. $\left(\operatorname{Re}_{2 i s}=1.5 \times 10^{5}, \mathrm{M}_{2 i s}=0.5\right.$, and $T_{w}=325^{\circ} \mathrm{K}$.) 
one additional local minimum and one additional local maximum in heat transfer parameters although it is less evident in the slight negative incidence case (i.e., $-17.7^{\circ}$ incidence). Generally, it can be concluded that the separated region always generates a redistribution in the heat flux by decreasing the value at the front separation region and by increasing it at the rear reattachment region. The higher or lower surface averaged effective value will depend on the particular geometry and conditions.

\subsection{Reynolds Number Effect}

Reynolds number effect is investigated by simulating different cases with the same incidence and exit Mach numbers but different fluid conditions so that the Reynolds number is changed. In order to achieve the required effect in the simulations, only the pressure level is modified. Figures 17 and 18 present the results when the Reynolds number is varied between 150,000 and 400,000 .

The dependence of heat flux on Reynolds number at separating and impingement regions can be obtained analytically on simple cases. At impingement points the case of plane and axisymetric laminar flows can be integrated to obtain the known dependence of the heat flux on the Reynolds number to the power of 0.5 [26]. Similarly, at separating points expansion equations can be obtained which also show a dependence on Reynolds number to the power of 0.5 in simple cases as wedge and Howarth's decelerating flow [27]. However, these methods are of difficult application to complex cases as presented here where a shear flow impinges on the wall with an inclination angle (i.e., the bubble reattachment occurring in our case), or where the separation occurs within a region of already separation bubble (i.e., the secondary bubble appearing in our case).

In our simulation cases, the HTC (i.e., heat flux) also increases with Reynolds number as expected. However, the maximum values at the reattachment point in these cases increases with an exponent approximately equal to 0.3 .

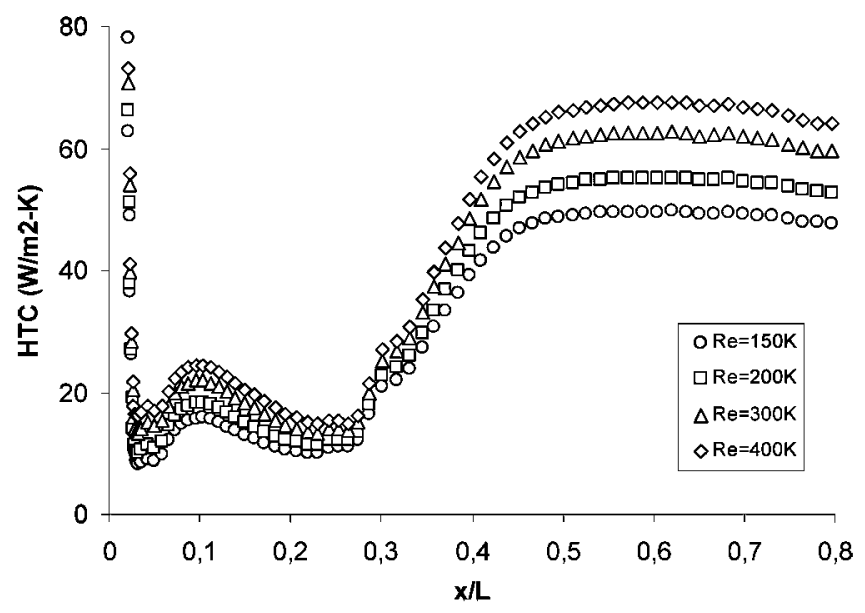

Figure 17. Heat transfer coefficient for different Reynolds number. $\left(\mathrm{M}_{2 i s}=0.5, \beta_{1}=90^{\circ}\right.$, and $T_{w}=325^{\circ} \mathrm{K}$. 


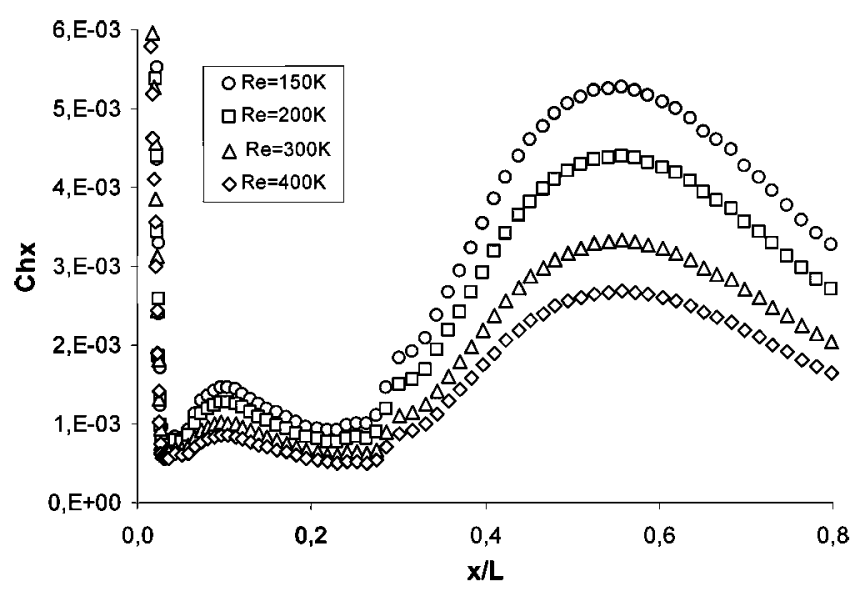

Figure 18. Stanton number for different Reynolds numbers. $\left(\mathrm{M}_{2 i s}=0.5, \beta_{1}=90^{\circ}\right.$, and $T_{w}=325^{\circ} \mathrm{K}$.)

It is interesting to note that the Stanton number varies inversely with the Reynolds number, as shown in Figure 18. By applying the definition relationship between HTC and Stanton number Ch in Eqs. (3) and (4), it can be seen that the ratio HTC/Ch must retain a dependence on Reynolds to the power of 1 . Therefore, the Stanton number dependence on the Reynolds number should vary with and exponent of -0.7 in these cases according to the exponent 0.3 found for the HTC, which is indeed confirmed by the maximum values at the reattachment point shown in Figure 18.

$$
\begin{gathered}
C h=\frac{H T C}{\rho_{e} C p U_{e}}=\frac{k}{L} \frac{1}{\operatorname{Pr}} \frac{1}{\operatorname{Re}_{L}} \mathrm{HTC} \\
\frac{\mathrm{HTC}}{\mathrm{Ch}}=\frac{L}{k} \operatorname{Pr} \operatorname{Re}_{L}
\end{gathered}
$$

One interesting feature is that, for all Reynolds numbers investigated, the size of the bubble and the internal structure (i.e., multiple bubble configuration) is the same, as can be concluded from the location of local maxima and minima in the figures. Although it could be expected that increasing the Reynolds number would reduce the size of the separation bubble, in this particular case the reattachment is driven by the acceleration of the flow and the role played by the Reynolds and the corresponding boundary layer instability and potential transition is expected to be very minor. This is a completely different behavior compared to cases in which there is no flow acceleration, and the reattachment is driven by boundary layer transition. In these latter cases, the increase of turbulence produces an early transition and reattachment and an increase in heat flux due to the stronger reattachment vortex, as has been shown by Hwang et al. [28] on a blunt flat plate subject to pulsating conditions.

Although the implemented numerical turbulence model was able to produce high turbulence and the corresponding boundary layer transition on the suction surface to avoid the back surface separation, at the pressure side the turbulence 
generation is concentrated on the external shear layer and from there it is convected downstream to the trailing edge and the downstream wake. Therefore, it is thought that in cases like this the turbulence is not a strong enough mechanism to force sufficient flow entrainment and perturbation to the shear layer to produce an early reattachment of the boundary layer, and it is then expected that the size of the separation bubble will depend weakly on the Reynolds number and turbulence.

\section{CONCLUSION}

A better understanding of the flow physics and the heat transfer mechanisms in large separated flow regions have been achieved by means of a numerical investigation on the T106-300 typical LPT airfoil subject to large negative incidence.

Flow separation is characterized by a pronounced reduction in HTC at the separation region, close to the leading edge where the minimum value is achieved, and by an increase at the reattachment region where the maximum value is achieved. Those are extreme values, much lower and higher than the ones obtained for attached flows. It is concluded that the velocity component perpendicular to the wall is the main contributor to the generation of ejection and impingement stagnation configurations, where the flow is taken from or towards the wall, hence affecting the thermal field in those regions and contributing to create a lower or higher temperature gradient at the wall and the corresponding HTC values. By analyzing the Reynolds-Colburn analogy all along the pressure side of the profile, the low coupling between the velocity component parallel to the wall and the thermal field and their gradients within the separation region is confirmed.

Additionally, it is also shown that an important variation in HTC values can occur within the separation region due to the presence of secondary separation bubbles which can create additional separation and reattachment points. This is confirmed by both numerical and experimental results for the high negative incidence (i.e., $-37.7^{\circ}$ incidence), which show the presence of one additional local maximum and one local minimum in HTC values that must indicate the presence of additional corresponding reattachment and separation points, hence indicating the presence of and the additional secondary separation bubble. Moreover, it is also shown by the numerical results that the multiple bubble configuration is found for all separated cases investigated here (i.e., negative incidence varying from $-17.7^{\circ}$ to $-37.7^{\circ}$ ).

Finally, the variation of the heat transfer with the Reynolds number is investigated. The numerical results show no variation of the separation bubble size with Reynolds number varying from 150,000 to 400,000 . A dependence of the HTC on the Reynolds number to the power of 0.3 is obtained in the separation region, in particular at the maximum value occurring at the main bubble reattachment point on the rear part of the separation bubble.

\section{REFERENCES}

1. M. Tutar and Ü. Sönmez, The Computation Modeling of Transitional Flow through a Transonic Linear Turbine: Comparative Performance of Various Turbulence Models, Numer. Heat Transfer A, vol. 58, pp. 403-427, 2010. 
2. V. K. Garg and A. A. Ameri, Comparison of Two-Equation Turbulence Models for Prediction of Heat Transfer on Film-Cooled Turbine Blades, Numer. Heat Transfer A, vol. 32, no. 4, pp. 347-371, 1997.

3. M. T. Schobeiri, B. Öztürk, M. Kegalj, and D. Bensing, On the Physics of Heat Transfer, and Aerodynamic Behavior of Separated Flow along a Highly Loaded Low Pressure Turbine Blade under Periodic Unsteady Wake Flow Varying of Turbulence Intensity, J. Heat Transfer, vol. 130, pp. 051703.1-051703.19, 2008.

4. J. C. Vogel and J. K. Eaton, Combined Heat Transfer and Fluid Dynamic Measurements Downstream of a Backward-Facing Step, J. Heat and Mass Transfer, vol. 107, pp. 922929, 1985.

5. E. M. Sparrow, S. S. Kang, and W. Chuck, Relation Between the Points of Flow Reattachment and Maximum Heat Transfer for Regions of Flow Separation, Int. J. Heat Mass Transfer, vol. 30, no. 7, pp. 1237-1246, 1987.

6. H. Kazeminejad, M. Ghamari, and M. A. Yaghoubi, A Numerical Study of Convective Heat Transfer from a Blunt Plate at Low Reynolds Number, Int. J. Heat Mass Transfer, vol. 39, no. 1, pp. 125-133, 1996.

7. G. H. Rhee, and H. J. Sung, A Low-Reynolds Number, Four Equation, Heat Transfer Model for Turbulent Separated and Reattaching Flows, Int. J. Heat Fluid Flow, vol. 18, pp. 38-44, 1997.

8. G. H. Rhee and H. J. Sung, Enhancement of Heat Transfer in Turbulent Separated and Reattaching Flow by Local Forcing, Numer. Heat Transfer A, vol. 37, pp. 733-753, 2000.

9. F. Bassi, S. Rebay, M. Savini, S. Colantuoni, and G. Santoriello, A Navier-Stokes Solver with Different Turbulence Models Applied to Film-Cooled Turbine Cascades, Heat Transfer and Cooling in Gas Turbines, Paper No. 41, AGARD-CP-527, 1993.

10. R. B. Rivir, J. P. Johnston, and J. K. Eaton, Heat Transfer on a Flat Surface under a Region of Turbulent Separation, J. Turbomachinery, vol. 116, pp 57-62, 1997.

11. R. J. Bellows and R. E. Mayle, Heat Transfer Downstream of a Leading Edge Separation Bubble, J. Turbomachinery, vol. 108, pp. 131-136, 1986.

12. P. De La Calzada and A. Alonso, Numerical Investigation of Heat Transfer in Turbine Cascades with Separated Flows, J. Turbomachinery, vol. 125, no. 2, pp. 260$266,2003$.

13. E. Lutum and F. Cottier, Aerothermal Predictions on a Highly Loaded Turbine Blade Including Effects of Flow Separation, Proc. European Turbomachinery Conference, vol. 2, pp. 1581-1591, Istambul, Turkey, 2011.

14. W. Merzkirch R. H. Page, and L. S. Fletcher, A Survey of Heat Transfer in Compressible Separated and Reattached Flows, AIAA J., vol. 26, no. 2, pp. 144-150, 1988.

15. F. Haselbach, and H. P. Schiffer, Aerothermal Investigations on Turbine Endwalls, and Blades (AITEB), Proc. ASME Turbo Expo 2004, paper no. GT2004-53078, Vienna, Austria, 2004.

16. A. Jameson, W. Schmidt, and E. Turkel, Numerical Solution of the Euler Equations by Finite Volume Method using Runge-Kutta Time Stepping Schemes, AIAA, Paper 81$1259,1981$.

17. R. Corral and J. Fernandez-Castañeda, Surface Mesh Generation by Means of Steiner Triangulation, Proc. 29th AIAA Fluid Dynamics Conference, vol. 39, pp. 176-180, Albuquerque, New Mexico, 1998.

18. D. C. Wilcox, Reassessment of the Scale Determining Equation for Advanced Turbulence Models, AIAA J., vol. 26, pp. 1299-1310, 1988.

19. R. Corral and J. Contreras, Quantitative Influence of the Steady Non-Reflecting Boundary Conditions on Blade-to-Blade Computations, Proc. 45th ASME Gas Turbine and Aeroengine Congress, Exposition and Users Symposium, ASME Paper 2000-GT-515, Munich, 2000. 
20. T. Arts and M. Lambert de Rouvroit, Aero-Thermal Performance of a Two Dimensional Highly Loaded Transonic Turbine Nozzle Guide Vane: A Test Case for Inviscid and Viscous Flow Computation, J. Turbomachinery, vol. 114, pp. 147-154, 1992.

21. A. Gehrer and H. Jericha, External Heat Transfer Predictions in a Highly Loaded Transonic Linear Turbine Guide Vane Cascade using and Upwind Biased Navier-Stokes Solver, J. Turbomachinery, vol. 121, pp. 525-531, 1999.

22. R. J. Boyle and A. A. Ameri, Grid Orthogonality Effects on Predicted Turbine Midspan Heat Transfer and Performance, J. Turbomachinery, vol. 119, pp. 31-38, 1997.

23. H. Hoheisel, Test Case E/CA-6, Subsonic Turbine Cascade T106, Test Cases for Computation of Internal Flows in Aero Engine Components, AGARD-AR-275, 1990.

24. E. J. Hall and J. D. Pletcher, Application of a Viscous-Inviscid Procedure to Predict Separated Flows with Heat Transfer, J. Heat Transfer, vol. 107, pp. 557-563, 1985.

25. P. R. Spalart and G. N. Coleman, Numerical Study of a Separation Bubble with Heat Transfer, Euro. J. Mech., B/Fluids, vol. 16, no. 2, pp. 169-189, 1997.

26. F. M. White, Viscous Fluid Flow, McGraw-Hill, pp. 162 and 248, 1991.

27. H. W. Kim and D. R. Jeng, Convective Heat Transfer in Laminar Boundary Layer Near the Separation Point, ASME Proc. of the 1988 National Heat Transfer Conference, vol. 3, pp. 471-476, 1988.

28. K. S. Hwang, H. J. Sung, and J. M. Hyun, Flow and Mass Transfer Measurements for a Flat Plate of Finite Thickness in Pulsating Flow, Int. J. Heat and Mass Transfer, vol. 41, pp. 2827-2836, 1998. 